\title{
Neural Correlates of Syntactic Processing in the Nonfluent Variant of Primary Progressive Aphasia
}

\author{
Stephen M. Wilson, ${ }^{1,2}$ Nina F. Dronkers, ${ }^{3,4}$ Jennifer M. Ogar, ${ }^{1}$ Jung Jang, ${ }^{1}$ Matthew E. Growdon, ${ }^{1}$ Federica Agosta, ${ }^{1,5}$ \\ Maya L. Henry, ${ }^{1}$ Bruce L. Miller, ${ }^{1}$ and Maria Luisa Gorno-Tempini ${ }^{1,6}$ \\ ${ }^{1}$ Memory and Aging Center, Department of Neurology, University of California, San Francisco, California 94143, ${ }^{2}$ Department of Speech, Language, and \\ Hearing Sciences, University of Arizona, Tucson, Arizona 85721, ${ }^{3}$ Center for Aphasia and Related Disorders, Veterans Administration Northern California \\ Health Care System, Martinez, California 94553, ${ }^{4}$ Department of Neurology, University of California, Davis, California 95817, ${ }^{5}$ Neuroimaging Research Unit, \\ Institute of Experimental Neurology, Division of Neuroscience, Scientific Institute and University Hospital San Raffaele, 20132 Milan, Italy, and ${ }^{6} \mathrm{Center}$ for \\ Mind/Brain Sciences, University of Trento, 38068 Rovereto, Italy
}

The left posterior inferior frontal cortex (IFC) is important for syntactic processing, and has been shown in many functional imaging studies to be differentially recruited for the processing of syntactically complex sentences relative to simpler ones. In the nonfluent variant of primary progressive aphasia (PPA), degeneration of the posterior IFC is associated with expressive and receptive agrammatism; however, the functional status of this region in nonfluent PPA is not well understood. Our objective was to determine whether the atrophic posterior IFC is differentially recruited for the processing of syntactically complex sentences in nonfluent PPA. Using structural and functional magnetic resonance imaging, we quantified tissue volumes and functional responses to a syntactic comprehension task in eight patients with nonfluent PPA, compared to healthy age-matched controls. In controls, the posterior IFC showed more activity for syntactically complex sentences than simpler ones, as expected. In nonfluent PPA patients, the posterior IFC was atrophic and, unlike controls, showed an equivalent level of functional activity for syntactically complex and simpler sentences. This abnormal pattern of functional activity was specific to the posterior IFC: the mid-superior temporal sulcus, another region modulated by syntactic complexity in controls, showed normal modulation by complexity in patients. A more anterior inferior frontal region was recruited by patients, but did not support successful syntactic processing. We conclude that in nonfluent PPA, the posterior IFC is not only structurally damaged, but also functionally abnormal, suggesting a critical role for this region in the breakdown of syntactic processing in this syndrome.

\section{Introduction}

Syntactic processing is a complex cognitive function whereby speakers and listeners implicitly construct and manipulate abstract hierarchical structures that specify the relationships between the words and morphemes that make up sentences (Gibson, 1998). Neuropsychological and functional neuroimaging studies have shown that brain regions throughout dominant perisylvian cortex are involved in syntactic processing (Bates et al., 1987a,b; Caplan and Hildebrandt, 1998; Dronkers et al., 2004; Tyler and Marslen-Wilson, 2008; Friederici et al., 2009). One region thought to be particularly important is the posterior infe-

Received May 17, 2010; revised Sept. 24, 2010; accepted 0ct. 18, 2010.

This work was supported by the National Institutes of Health [National Institute on Deafness and Other Communication Disorders Grant R03 DC010878 to S.M.W., National Institute of Neurological Disorders and Stroke Grant R01 NS050915 to M.L.G.-T., and National Institute on Aging Grants P50 AG03006 and P01 AG019724 to B.L.M.]; State of California (DHS 04-35516); Alzheimer's Disease Research Center of California (03-75271 DHS/ADP/ARCC); Larry L. Hillblom Foundation; John Douglas French Alzheimer's Foundation; Koret Family Foundation; and McBean Family Foundation. We thank Laura Calverley for drawing the stimulus pictures, Max Besbris, Victor Laluz, Paul Keselman, Lara Stables, and Adam Gazzaley for assistance with functional imaging, Kate Rankin and Judy Pa for helpfu discussions, two anonymous reviewers for their constructive comments, all of the members of the UCSF Memory and Aging Center who contributed to patient evaluation and care, and all of the patients, caregivers, and volunteers for their participation in our research.

Correspondence should be addressed to Stephen M. Wilson, Department of Speech, Language, and Hearing Sciences, University of Arizona, P.0. Box 210071, Tucson, AZ 85721-0071. E-mail: smwilson@u.arizona.edu.

DOI:10.1523/JNEUROSCI.2547-10.2010

Copyright $\odot 2010$ the authors $\quad 0270-6474 / 10 / 3016845-10 \$ 15.00 / 0$ rior frontal cortex (IFC), which we define as including the pars opercularis and triangularis of the inferior frontal gyrus, and the adjacent inferior frontal sulcus. Patients with damage encompassing this area often present with Broca's aphasia, which is characterized by expressive and receptive agrammatism (Caramazza and Zurif, 1976; Goodglass, 1993), and numerous functional imaging studies have revealed increased inferior frontal activation for syntactically complex sentences relative to simpler ones (Stromswold et al., 1996; Caplan et al., 1999).

Primary progressive aphasia (PPA) is a clinical syndrome in which progressive speech and/or language deficits are associated with degeneration of dominant hemisphere language regions (Mesulam, 2001). In particular, degeneration of inferior frontal cortex has been associated with the nonfluent/agrammatic variant of PPA, in which syntactic deficits are prominent (Gorno-Tempini et al., 2004). Nonfluent PPA patients produce agrammatic speech (Hodges and Patterson, 1996; Thompson et al., 1997; Weintraub et al., 2009), are impaired in comprehending syntactically complex sentences (Hodges and Patterson, 1996; Grossman and Moore, 2005), and are relatively insensitive to grammatical violations (Grossman et al., 2005; Cotelli et al., 2007). Structural imaging studies using voxel-based morphometry have demonstrated associations between left inferior frontal volume loss and both receptive (Amici et al., 2007; Peelle et al., 2008) and expressive (Wilson et al., 2010) syntactic deficits. 
Unlike in stroke-induced Broca's aphasia, in which the posterior IFC and surrounding regions are typically completely destroyed, atrophy is gradual and progressive in PPA. Since functional and structural changes in neurodegenerative disease do not necessarily correspond directly (Dickerson and Sperling, 2009), this raises the question of to what extent surviving neural tissue in this region is functional. Metabolic studies have shown hypometabolism of inferior frontal regions in nonfluent PPA (Nestor et al., 2003, Rabinovici et al., 2008), but little is known regarding task-related modulation of functional activity by syntactic processing. One study of three nonfluent PPA patients scanned during a sentence comprehension task showed reduced left inferior frontal activity, but the small sample size did not permit a direct comparison between patients and controls (Cooke et al., 2003).

In this study, our primary aim was to determine whether the atrophic left posterior IFC is differentially recruited for the processing of syntactically complex sentences in nonfluent PPA, as it is in normal subjects. We approached this question by using structural and functional magnetic resonance imaging to quantify regional changes in tissue volume and functional responses to a syntactic comprehension task in patients with nonfluent PPA, in comparison to healthy age-matched controls. More generally, we compared the spatial distribution throughout the brain of structural and functional changes, including potentially compensatory functional activity.

\section{Materials and Methods}

Participants. We successfully scanned eight patients with nonfluent PPA and 24 normal controls over an 18 month period. Patients and normal control subjects were recruited through the Memory and Aging Center at the University of California, San Francisco (UCSF). All participants gave written informed consent, and the study was approved by the Committee on Human Research at UCSF. Patients and controls received a comprehensive multidisciplinary evaluation including neurological history and examination, neuropsychological testing, and neuroimaging.

A diagnosis of PPA required progressive deterioration of speech and/or language functions, and that deficits be largely restricted to speech and/or language for at least 2 years (Mesulam, 2001). Patients were diagnosed with the nonfluent variant of PPA based on new consensus guidelines (Gorno-Tempini et al., 2011). The nonfluent variant criteria require the presence of one or both of two core features: agrammatism and/or effortful speech. Additionally, at least two of three supporting features must be present: comprehension deficits for syntactically complex sentences, spared single-word comprehension, and/or spared object knowledge. Neuroimaging results were not used for diagnostic purposes, but only to rule out other causes of focal brain damage.

Additional inclusion criteria were fluency in English and a Mini-Mental State Examination score of at least 15 . Nine patients met these criteria and were scanned, but one was excluded because she performed at chance on all conditions, including those that required lexical knowledge alone (see below), so all analyses were based on the remaining eight patients.

Two of the eight patients were severely agrammatic in their speech production (e.g., "and uh a blanket... and ... a thongs off the man ... and um ... uh . . . teenagers um . . . in the kite"), two were moderately so (e.g., "the family is have a picnic, and um, the young son is flying their kei- k- kite"), one was near-mute with severely agrammatic written language (e.g., "man read book girl the coffee in cup"), one was near-mute with moderately agrammatic written language (e.g., "the couple having a picnic, they are sitting a blanket under a tree"), and two had primarily speech motor deficits, with intact syntax in production (e.g., "the fellow is reading a book, the woman is pouring some l- liquids"), and mild syntactic deficits evident only in comprehension of complex sentences. All patients were clinically diagnosed with apraxia of speech, with severity ranging from 2 to 7 on a seven-point scale (Wertz et al., 1984), and four of the eight were dysarthric (including the two who were near-mute).
Table 1. Demographic, clinical, and neuropsychological characteristics of patients and controls

\begin{tabular}{|c|c|c|}
\hline Variable & Controls & Nonfluent PPA \\
\hline \multicolumn{3}{|l|}{ Demographic } \\
\hline Age & $66.8 \pm 4.2$ & $69.3 \pm 7.3$ \\
\hline $\operatorname{Sex}(M / F)$ & $7 / 17$ & $2 / 6$ \\
\hline Handedness (R/L) & $22 / 2$ & $8 / 0$ \\
\hline Education (years) & $17.2 \pm 2.0$ & $16.3 \pm 2.9$ \\
\hline \multicolumn{3}{|l|}{ Clinical } \\
\hline Mini Mental Status Examination (30) & $29.3 \pm 0.8$ & $25.7 \pm 3.1^{*}$ \\
\hline Clinical Dementia Rating & $\mathrm{N} / \mathrm{A}$ & $0.4 \pm 0.4^{t \dagger}$ \\
\hline Clinical Dementia Rating (sum of boxes) & $\mathrm{N} / \mathrm{A}$ & $2.3 \pm 2.4$ \\
\hline Age at disease onset & $\mathrm{N} / \mathrm{A}$ & $63.8 \pm 6.7$ \\
\hline Years from first symptom & $\mathrm{N} / \mathrm{A}$ & $5.5 \pm 2.8$ \\
\hline \multicolumn{3}{|l|}{ Language production } \\
\hline Confrontation naming (BNT, 15) & $14.5 \pm 0.7$ & $11.6 \pm 3.7^{*}$ \\
\hline Phonemic fluency (D words in $1 \mathrm{~min}$ ) & $17.6 \pm 4.1$ & $6.7 \pm 3.6^{* \dagger}$ \\
\hline Semantic fluency (Animals in $1 \mathrm{~min}$ ) & $23.8 \pm 4.3$ & $11.2 \pm 5.5^{* \dagger}$ \\
\hline Speech fluency $(W A B, 10)$ & $10.0 \pm 0.0^{\ddagger}$ & $4.9 \pm 3.5^{*}$ \\
\hline Apraxia of speech rating (MSE, 7) & $\mathrm{N} / \mathrm{A}$ & $3.8 \pm 2.1^{*}$ \\
\hline Dysarthria rating $(M S E, 7)$ & $\mathrm{N} / \mathrm{A}$ & $2.5 \pm 3.1^{*}$ \\
\hline Repetition (WAB, 100) & $99.5 \pm 0.9^{\neq}$ & $81.3 \pm 10.5^{* \dagger}$ \\
\hline \multicolumn{3}{|l|}{ Language comprehension } \\
\hline Auditory word recognition (PPVT, 16) & $15.7 \pm 0.7$ & $13.6 \pm 2.7^{*}$ \\
\hline Sequential commands (WAB, 80) & $80.0 \pm 0.0^{\ddagger}$ & $71.6 \pm 8.4^{*}$ \\
\hline Semantic knowledge (PPT-P, 52) & $51.8 \pm 0.4^{\ddagger}$ & $49.0 \pm 2.9$ \\
\hline \multicolumn{3}{|l|}{ Visuospatial function } \\
\hline Modified Rey-0sterrieth copy (17) & $15.0 \pm 1.1$ & $14.6 \pm 2.4$ \\
\hline \multicolumn{3}{|l|}{ Visual memory } \\
\hline Modified Rey-0sterrieth delay (17) & $11.7 \pm 2.3$ & $9.4 \pm 3.4$ \\
\hline \multicolumn{3}{|l|}{ Verbal memory } \\
\hline CVLT-MS trials 1-4 (40) & $28.7 \pm 3.1^{\neq}$ & $21.4 \pm 7.9^{*}$ \\
\hline CVLT-MS 30 s free recall (10) & $7.9 \pm 1.6^{\ddagger}$ & $6.4 \pm 2.4$ \\
\hline CVLT-MS 10 min free recall (10) & $7.3 \pm 1.6^{\ddagger}$ & $0.1 \pm 0.4^{*}$ \\
\hline \multicolumn{3}{|l|}{ Executive function } \\
\hline Digit span backwards & $5.6 \pm 1.2$ & $3.4 \pm 1.3^{*}$ \\
\hline Modified trails (lines per minute) & $37.8 \pm 12.6$ & $19.3 \pm 11.8$ \\
\hline Calculation (5) & $4.8 \pm 0.4$ & $4.9 \pm 0.4$ \\
\hline
\end{tabular}

Values are means \pm SD. Variables were compared between patients and controls using $t$ test with unequal variance where appropriate, the Wilcoxon signed-rank test for measures with floor or ceiling effects, and Fisher's exact test for discrete variables. *Significantly impaired relative to controls, $p<0.05$. 'Excluding two patients who were nearly mute. ${ }^{\ddagger}$ Data from Gorno-Tempini et al. (2004) since present control group was not tested on these variables. ${ }^{\ddagger \ddagger}$ The Clinical Dementia Rating was 0 for 3 patients, 0.5 for 3 patients, and 1 for 2 patients. BNT, Boston Naming Test; WAB, Western Aphasia Battery; MSE, Motor Speech Evaluation; PPVT, Peabody Picture Vocabulary Test; PPT-P, Pyramids and Palm Trees_-Pictures; CVLT-MS, California Verbal Learning Test-Mental Status. See Kramer et al. (2003) for detailed description of neuropsychological testing procedures and Gorno-Tempini et al. (2004) for detailed description of language testing procedures.

Demographic, clinical, and neuropsychological characteristics for all participants are provided in Table 1 . There were no significant differences between patients and controls in age, sex, handedness, or education.

In addition to the 24 normal controls who took part in functional imaging, structural images from another group of 50 healthy agematched controls were used to create a template for intersubject normalization and voxel-based morphometry.

Experimental design. Participants were scanned with functional MRI as they listened to sentences and selected the matching picture from two choices: a target and a foil. Seven conditions that varied in terms of the syntactic processing required (see below) were presented in a block design. All conditions required sentence comprehension; we did not use any low-level control conditions such as backwards sentences or signal-correlated noise, since in pilot studies we had found these to be confusing for some patients. There were three blocks per condition, for a total of 21 blocks, presented in random order. Each block was $28 \mathrm{~s}$ in length and contained four equally spaced trials, and there were $16 \mathrm{~s}$ rest periods between blocks and at the beginning and end of the experiment. The total duration of the functional sequence was $940 \mathrm{~s}$. 


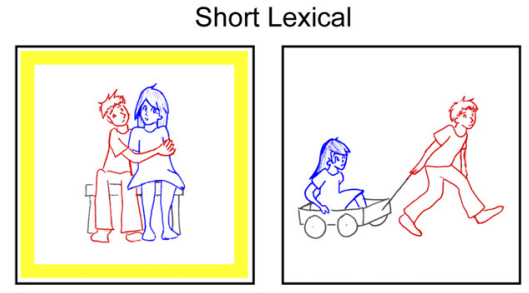

The boy is hugging the girl.

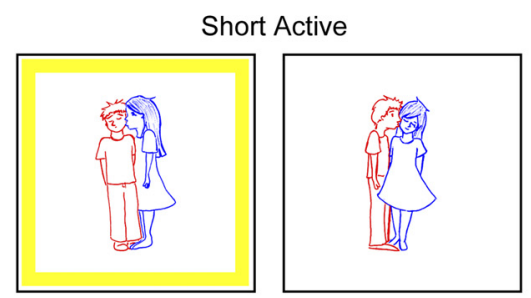

The girl is kissing the boy.

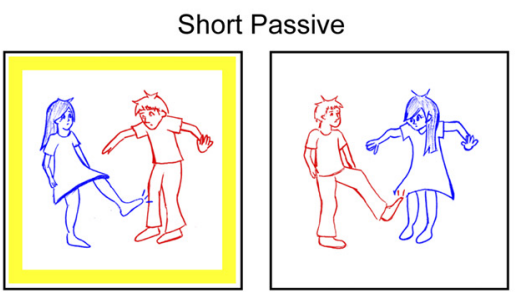

The boy is kicked by the girl.

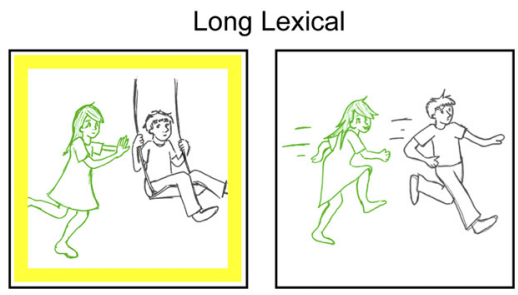

The girl who is green is pushing the boy.

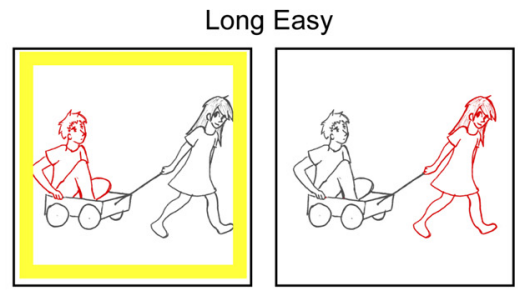

The girl is pulling the boy who is red.

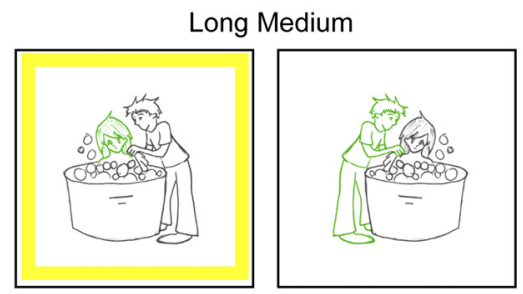

The girl who is washed by the boy is green.

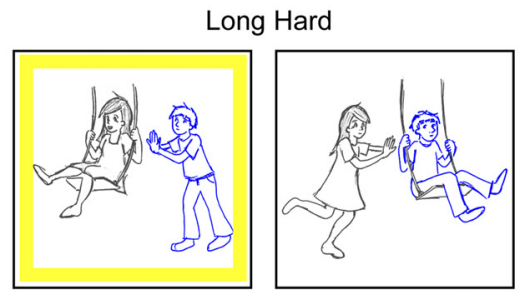

The boy who the girl is pushed by is blue.

Figure 1. Experimental design. There were seven conditions that varied in terms of the syntactic processing required. The targets are shown on the left, surrounded by a yellow box. In the actual experiment, targets and foils were presented randomly on the left or right, and the yellow box appeared to indicate the subject's choice.

Each trial began with the presentation of two pictures: one on the left and one on the right of the screen. One second later, a sentence was presented auditorily that matched one of the two pictures. Participants selected the matching picture at any point (either before or after hearing the end of the sentence) by pressing one of two buttons. A yellow box then appeared around the chosen picture. If participants failed to respond within $5 \mathrm{~s}$ from the onset of sentence presentation, the pictures disappeared; however, late responses were still recorded and analyzed. One second later, the next trial began.

The extent to which a sentence comprehension task makes demands on syntactic processing resources depends not only on the structure of the sentences, but also on the nature of the foil pictures. For instance, if the foil picture involves a different action than the target picture, a correct response can be generated based on lexical information alone (the verb), without necessarily even parsing the sentence. On the other hand, if the foil picture contains the same actors as the target picture yet their thematic roles (i.e., agent, patient) are different, it is necessary to attend to syntactic structure to determine the correct role assignments and make the correct choice. In cases where syntactic structure determines the correct response, there is a further important difference between sentences with canonical and noncanonical structures. A sentence such as "The boy kissed the girl" is canonical in every respect: the agent is en- coded as the subject and immediately precedes the verb, and the patient is encoded as the object and immediately follows the verb. These configurations are prototypical in English. In contrast, "The girl was kissed by the boy" is noncanonical is several ways: the patient is encoded as the subject and therefore precedes the verb, whereas the agent is encoded in an adjunct "by" phrase and so follows the verb. While theoretical explanations differ, it is well established that noncanonical structures are more difficult and require more resources to process than canonical structures, as indexed by accuracy and/or reaction time (Bates and MacWhinney, 1989; Caplan and Hildebrandt, 1998; Gibson, 1998).

With these considerations in mind, we devised seven conditions that differed in terms of whether or not syntactic information was necessary to respond correctly, and if so, whether the syntactic structure of the sentence was canonical or noncanonical (Fig. 1, Table 2). Three conditions consisted of short sentences ( 7 syllables each), and four consisted of long sentences (10 syllables each). The short conditions contained just one sentence type each (12 items), whereas the long conditions contained two different sentence types (6 items of each) determined in pilot testing to be similar in difficulty. Within each length category, all conditions were matched for length, for lexical content, and for the point at which the sentence would disambiguate between the target and foil pictures.

To keep lexical demands to a minimum, all sentences were constructed using just two highfrequency nouns (boy, girl), one of seven highfrequency verbs (push, pull, kiss, kick, chase, wash, hug), and for the long sentences, one of three high-frequency color adjectives (red, green, blue). The stimuli were digitally recorded by one of the authors (M.E.G.). Many of the sentences and pictures were based loosely on items from the Curtiss-Yamada Comprehensive Language Evaluation (S. Curtiss and J. Yamada, unpublished test), though our experimental design differed from this test in many ways.

The primary contrast of interest, for both behavioral and imaging analyses, was between conditions with noncanonical sentences (short passive, long medium, and long hard) and those with canonical sentences (short lexical, short active, long lexical, and long easy). The short passive condition was multiplied by two to balance short and long conditions across the contrast. This contrast incorporated both short and long conditions, averaging the canonicity contrast across them. Our design did not permit the direct comparison of short and long conditions, because they differed in sentence length, lexical content, and point of disambiguation. A secondary contrast of interest was the average of all seven conditions.

Neuroimaging protocol. Before scanning, participants were trained on the task until they could perform it confidently for the short lexical condition. They then lay supine in a Siemens 3 tesla Trio scanner. They wore earplugs and padded headphones, viewed a monitor through a mirror, and held a fiber-optic response pad in their right hand, with their index and middle fingers on the left and right buttons, respectively.

For intersubject registration and voxel-based morphometry, a T1weighted 3D magnetization prepared rapid acquisition gradient echo (MPRAGE) sequence was acquired with the following parameters: 160 sagittal slices; slice thickness $=1 \mathrm{~mm}$; field of view $=256 \times 256 \mathrm{~mm}$; matrix $=230 \times 256$; repetition time $(\mathrm{TR})=2300 \mathrm{~ms}$; echo time $(\mathrm{TE})=$ $2.98 \mathrm{~ms}$; flip angle $=9^{\circ}$. 
Table 2. Sentence structures and foils in the seven conditions

\begin{tabular}{|c|c|c|c|}
\hline Condition & Sentence example & Foil picture & Syntactic processing required \\
\hline Short lexical & The boy is hugging the girl & Different action (e.g., pulling) & Can be decided based on lexical information alone \\
\hline Short active & The girl is kissing the boy & Agent and patient reversed & $\begin{array}{l}\text { Relatively straightforward, since sentence } \\
\text { conforms to language-general word order } \\
\text { principles }\end{array}$ \\
\hline Short passive (noncanonical) & The boy is kicked by the girl & Agent and patient reversed & $\begin{array}{l}\text { Noncanonical word order (patient is initial and } \\
\text { agent is in prepositional phrase), so requires } \\
\text { attention to passive morphosyntax }\end{array}$ \\
\hline \multirow[t]{2}{*}{ Long lexical } & The girl who is green is pushing the boy & Different action (e.g., chasing) & Can be decided based on lexical information alone \\
\hline & The boy is kissing the girl who is red & Different-colored patient (e.g., green girl) & Can be decided based on lexical information alone \\
\hline \multirow[t]{2}{*}{ Long easy } & The girl is pulling the boy who is red & $\begin{array}{l}\text { Color is assigned to wrong participant (e.g., red } \\
\text { girl) }\end{array}$ & $\begin{array}{l}\text { Relatively straightforward, since correct head noun } \\
\text { for relative clause is in canonical position } \\
\text { immediately to the left of the relative }\end{array}$ \\
\hline & The boy who is red is chasing the girl & Agent and patient reversed & $\begin{array}{l}\text { Relatively straightforward, since sentence } \\
\text { conforms to language-general word order } \\
\text { principles }\end{array}$ \\
\hline \multirow[t]{2}{*}{ Long medium (Noncanonical) } & The girl who is washed by the boy is green & $\begin{array}{l}\text { Color is assigned to wrong participant (e.g., } \\
\text { green boy) }\end{array}$ & $\begin{array}{l}\text { Passive relative clause separates adjective from the } \\
\text { noun it modifies; attention to hierarchical } \\
\text { structure is required to assign the adjective to } \\
\text { the matrix subject, not the adjacent noun }\end{array}$ \\
\hline & The girl who the boy is hugging is red & Agent and patient reversed & $\begin{array}{l}\text { Object relative clause results in noncanonical word } \\
\text { order (the agent follows the patient), so } \\
\text { requires attention to the syntax of the relative } \\
\text { clause }\end{array}$ \\
\hline \multirow[t]{2}{*}{ Long hard (Noncanonical) } & The boy who the girl is pushed by is blue & Agent and patient reversed & $\begin{array}{l}\text { Highly infrequent adjunct relative passive clause } \\
\text { make assignment of thematic roles very } \\
\text { difficult }\end{array}$ \\
\hline & The girl who the boy is kissed by is green & $\begin{array}{l}\text { Color is assigned to wrong participant (e.g., } \\
\text { green boy) }\end{array}$ & $\begin{array}{l}\text { Passive adjunct relative clause separates adjective } \\
\text { from the noun it modifies; attention to hierarchical } \\
\text { structure is required to assign the adjective to the } \\
\text { matrix subject, not the closer noun }\end{array}$ \\
\hline
\end{tabular}

Italicized words in the examples show which word disambiguates the sentence between the target and foil pictures. Note that for each of the four long conditions, there were two different types of items.

For the blood oxygen level-dependent (BOLD) functional MRI paradigm, $470 \mathrm{~T} 2^{\star}$-weighted echo-planar volumes were acquired with the following parameters: $32 \mathrm{AC} / \mathrm{PC}$-aligned axial slices in interleaved order; slice thickness $=3.6 \mathrm{~mm}$ with $0.9 \mathrm{~mm}$ gap; field of view $=230 \times 230$ $\mathrm{mm}$; matrix $=96 \times 96 ; \mathrm{TR}=2000 \mathrm{~ms} ; \mathrm{TE}=28 \mathrm{~ms}$; flip angle $=90^{\circ}$.

Auditory and visual stimuli were presented and accuracy and reaction time data were recorded with PsychToolbox 3.0.8 (Brainard, 1997; Pelli, 1997) running under MATLAB 7.4 (Mathworks). Before the functional scan, additional practice trials were presented in the scanner while functional data were acquired (and discarded), to familiarize subjects with performing the task in the scanner environment, and also to adjust the volume of the stimuli to a comfortable level at which sentences could be heard over the background scanner noise.

Analysis of behavioral data. Accuracy and reaction time were compared across groups using $t$ tests with unequal variance in JMP (SAS Institute). Reaction time was measured from the onset of the first word of the sentence that disambiguated between the target and foil pictures.

Analysis of structural imaging data. Structural T1 images were corrected for bias field, segmented into gray matter, white matter, and CSF, and initially normalized to Montreal Neurological Institute (MNI) space using the Unified Segmentation procedure (Ashburner and Friston, 2005) implemented in SPM5 (Friston et al., 2007), running under MATLAB 7.4. More anatomically precise intersubject registration was then performed with the Diffeomorphic Anatomical Registration Through Exponentiated Lie algebra (DARTEL) toolbox (Ashburner, 2007) by warping each subject's image to a template created from the 50 additional normal control subjects.

To identify regions of significant atrophy in the group of nonfluent PPA patients, we summed Jacobian-modulated gray matter and white matter images to create maps of brain parenchyma. These images were smoothed with a $12 \mathrm{~mm}$ full-width at half-maximum (FWHM) Gaussian kernel. The nonfluent PPA group was compared to 74 normal controls (the 24 normal control subjects who took part in the functional study, plus the 50 additional subjects), covarying out age, sex, and total intracranial volume. Percentage volume loss was plotted for regions with at least $15 \%$ volume loss.

Analysis of functional imaging data. The functional MRI data for each subject were preprocessed with standard methods in SPM5. Data were corrected for slice timing differences, realigned to account for withinscan head movement, smoothed with a Gaussian kernel of $8 \mathrm{~mm}$ FWHM, and high-pass filtered (cutoff $=128 \mathrm{~s}$ ) to remove slow signal drift.

In our primary analysis, we examined signal change as a function of condition. The design matrix contained one explanatory variable for each of the seven conditions, which consisted of a boxcar function convolved with a hemodynamic response function (HRF). Additional covariates of no interest were included to reduce error variance: three translation and three rotation parameters (saved during realignment), and raw signal time courses from a white matter region of interest (ROI), a CSF ROI, and the whole-brain global signal. All raw data were manually examined and volumes where there was excessive head motion (visible interleaving artifact) or other artifacts were excluded. The number of volumes excluded was $26.5 \pm 22.5$ in nonfluent PPA patients and $11.5 \pm$ 15.9 in controls. We fit a general linear model to the BOLD signal time course for each voxel in each participant using Restricted Maximum Likelihood and an autoregressive AR(1) model to correct for nonsphericity arising from serial correlations. The main contrast of interest was between noncanonical and canonical conditions, as described above. We refer to regions activated by this contrast as "modulated by syntactic complexity." A secondary contrast of interest was all conditions relative to rest.

Random effects analyses were performed on contrast images from individual subjects, which were normalized to MNI space by applying the transformations derived with Unified Segmentation and DARTEL described above. Patients and controls were compared with $t$ tests with unequal variance. Contrasts identifying regions activated less in patients than controls were masked to include only regions activated 

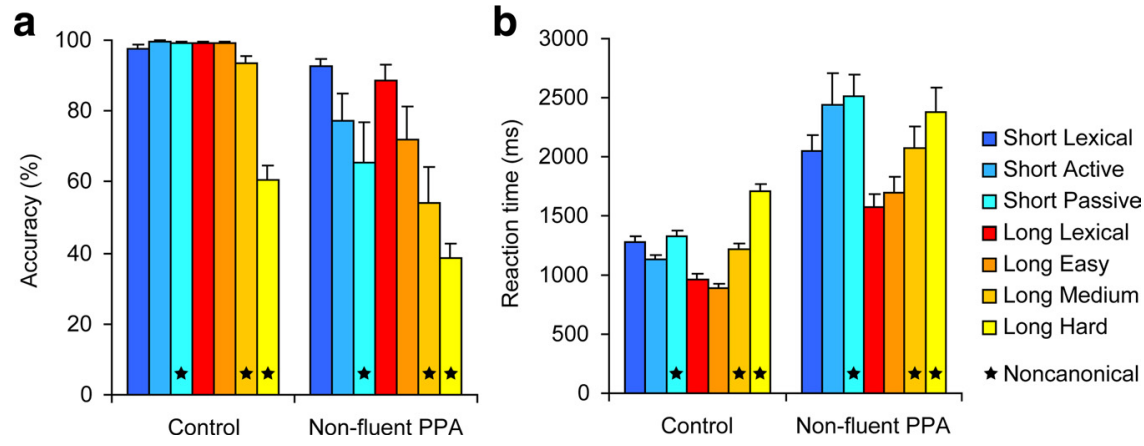

Figure 2. Behavioral data recorded during scanning. $\boldsymbol{a}, \boldsymbol{b}, \operatorname{Accuracy}(\boldsymbol{a})$ and reaction time $(\boldsymbol{b})$ in each of the seven conditions, in controls and nonfluent PPA patients. Noncanonical conditions are indicated by the star symbol. Error bars show SEM.
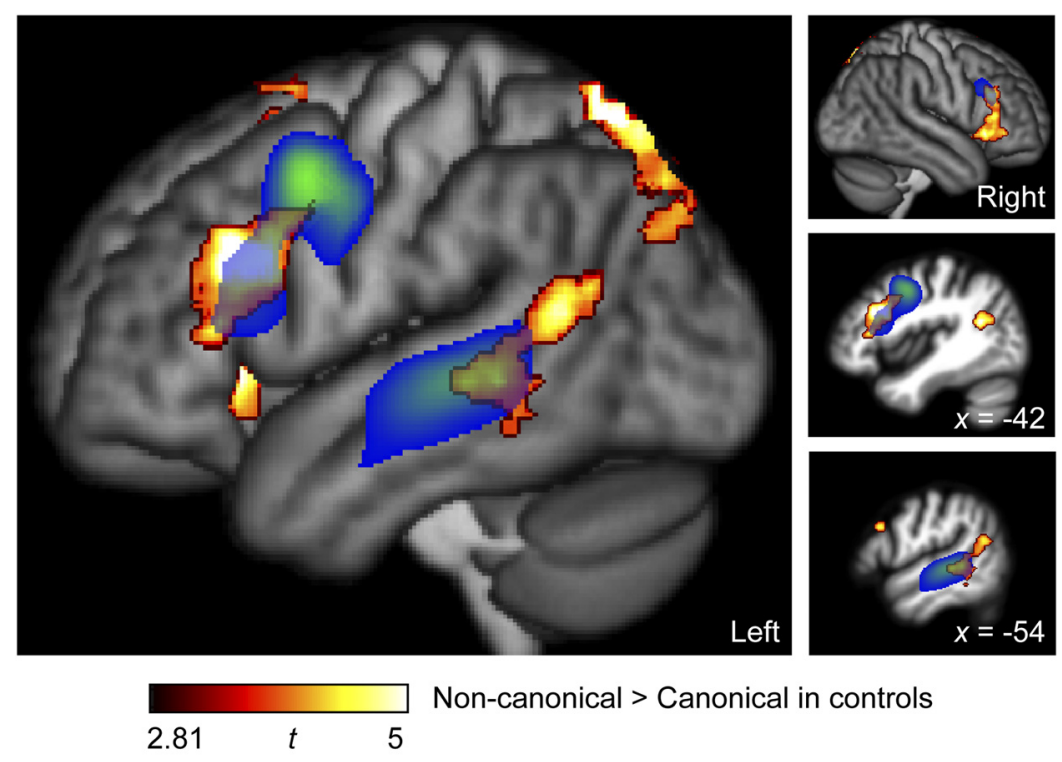

Non-canonical > Canonical in controls

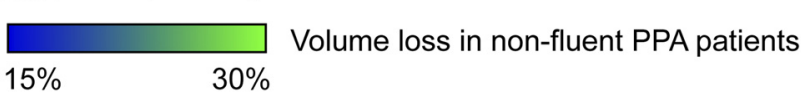

Figure 3. Overlap between regions modulated by syntactic complexity in controls and atrophic regions in nonfluent PPA. Regions activated for noncanonical versus canonical sentences in normal controls ( $n=24$ ) (hot) and regions with volume loss of $15 \%$ or greater in patients $(n=8)$ (blue-green). Lateral renderings of each hemisphere and two sagittal slices through the left hemisphere are shown.

Table 3. Atrophic brain regions in nonfluent PPA patients

\begin{tabular}{lrrrrrr}
\hline & \multicolumn{3}{l}{ MNI coordinates } & & $\begin{array}{l}\text { Volume } \\
\left(\mathrm{mm}^{3}\right)\end{array}$ & $\begin{array}{l}\text { Max } \\
\text { atrophy }\end{array}$ \\
\cline { 2 - 4 } Brain region & \multicolumn{1}{c}{$x$} & $y$ & $z$ & & 21,128 & $29 \%$ \\
\hline $\begin{array}{l}\text { Left precentral gyrus, IFS, and } \\
\quad \text { dorsal posterior IFG }\end{array}$ & -34 & 5 & 39 & & \\
$\begin{array}{l}\text { Left STG, STS, and MTG } \\
\text { Right IFS }\end{array}$ & -54 & -29 & -4 & 8768 & $24 \%$ \\
\hline
\end{tabular}

MNI coordinates are centers of mass.

in controls, and corrected only for the volume of those regions. Contrasts identifying regions activated more in patients than controls were masked to include only regions activated in patients. All masks were thresholded at voxelwise $p<0.005$. All statistical maps were thresholded at voxelwise $p<0.005$, and then corrected for multiple comparisons at $p<0.05$ based on cluster extent according to Gaussian random field theory.

We further examined signal change as a function of condition in several left perisylvian ROIs. Anterior and posterior perisylvian regions were selected because of their known involvement in syntactic processing (Bates et al., 1987a,b; Caplan and Hildebrandt, 1998; Dronkers et al.,
2004; Tyler and Marslen-Wilson, 2008; Friederici et al., 2009). ROIs were defined based on functional contrasts and structural changes in patients, as specified in the Results. Signal change for each condition was averaged across all voxels in the ROI, using custom MATLAB scripts. Signal differences were compared between groups with $t$ tests with unequal variance using JMP.

In each ROI, we also examined whether there were relationships between atrophy and both functional contrasts of interest, i.e., modulation by syntactic complexity, and overall activity relative to rest. Atrophy was quantified by subtracting from 1 the mean smoothed Jacobian-modulated gray matter and white matter estimates (corrected for total intracranial volume) averaged over the ROI, and correlations between atrophy and functional measures were calculated with JMP.

A challenge in comparing patients and healthy controls in imaging studies of impaired domains is that patients typically differ from controls in task performance as quantified by accuracy and reaction time (Price et al., 2006). To address this challenge, two ancillary analyses were performed in which we compared nonfluent PPA patients and controls while matching performance insofar as that was possible.

In the first ancillary analysis, we quantified modulation of signal by syntactic complexity by using reaction time as a proxy for allocation of syntactic processing resources (Wilson et al., 2009), instead of defining syntactic complexity in terms of noncanonical versus canonical conditions. Only correct trials were considered, and no trials were included from conditions on which participants scored $<8$ out of 12 correct, because correct trials in such conditions may well have just been guesses. The four trials within each block were treated as separate events with a duration of $4 \mathrm{~s}$ each, convolved with an HRF. There were two separate explanatory variables for short and long correct trials, each of which was parametrically modulated by another variable coding reaction time for that trial. Reaction time was demeaned based on the mean of all short or long correct trials, and clipped at 1.5 SDs from the mean. There were two additional explanatory variables modeling incorrect trials and trials on which the participant did not respond. The same covariates of no interest were included as in the main analysis. The contrast of interest was signal change per second of reaction time, which was averaged across the short and long parametric variables. Patients and controls were compared as described above.

In the second ancillary analysis, we compared signal change relative to rest between patients and controls, on a subset of blocks that were matched for accuracy and reaction time across the two groups. We selected the most advanced long condition on which each nonfluent PPA patient scored at least 8 out of 12 correct (long lexical for 4 subjects, long easy for 1 subject, and long medium for 3 subjects). We then selected 10 long blocks from normal controls (long medium for 4 subjects, long hard for 6 subjects) such that neither accuracy $\left(t_{(12.46)}=0.14, p=0.89\right)$ nor reaction time $\left(t_{(9.02)}=1.28, p=0.23\right)$ differed between patients and controls for the blocks chosen. Contrast images from the primary analysis described above were submitted to a $t$ test with unequal variance, with accuracy and reaction time included as covariates of no interest. Patients and controls were compared as described above. 
Table 4. Brain regions modulated by syntactic complexity in controls

\begin{tabular}{|c|c|c|c|c|c|c|}
\hline \multirow[b]{2}{*}{ Brain region } & \multicolumn{3}{|c|}{ MNI coordinates } & \multirow[b]{2}{*}{ Volume $\left(\mathrm{mm}^{3}\right)$} & \multirow[b]{2}{*}{ Maxt } & \multirow[b]{2}{*}{$p$} \\
\hline & $x$ & $y$ & $Z$ & & & \\
\hline Left IFS, dorsal posterior IFG, and insula & -40 & 21 & 20 & 9400 & 5.72 & $<0.001$ \\
\hline IFS & -46 & 22 & 30 & & 5.72 & \\
\hline Anterior insula & -30 & 22 & 0 & & 5.49 & \\
\hline Right IFS, dorsal posterior IFG, and insula & 45 & 26 & 7 & 5432 & 4.95 & 0.001 \\
\hline IFS & 48 & 26 & 26 & & 4.65 & \\
\hline Anterior insula & 36 & 22 & -4 & & 4.95 & \\
\hline Left mid-posterior STS and adjacent MTG & -51 & -48 & 9 & 5016 & 5.46 & 0.001 \\
\hline Posterior STS & -44 & -54 & 18 & & 5.46 & \\
\hline Mid-STS & -50 & -34 & -2 & & 3.73 & \\
\hline Left precuneus and superior parietal cortex & -15 & -72 & 53 & 4856 & 7.22 & 0.001 \\
\hline Bilateral supplementary motor area & 1 & 15 & 56 & 3552 & 4.81 & 0.009 \\
\hline Left precuneus & -4 & -58 & 42 & 2560 & 4.73 & 0.049 \\
\hline
\end{tabular}

MNI coordinates for clusters are centers of mass, whereas MNI coordinates for multiple regions within a cluster are local maxima.

\section{Results}

\section{Behavioral data}

Nonfluent PPA patients performed less accurately overall $(69.8 \pm 14.0 \%)$ than controls $\left(92.8 \pm 3.1 \% ; t_{(7.23)}=-4.59, p=\right.$ 0.0023 ), and they performed disproportionately worse on more syntactically complex (noncanonical) conditions relative to less complex (canonical) conditions $\left(t_{(8.23)}=\right.$ $-4.04, p=0.0035$ ) (Fig. 2a).

Nonfluent PPA patients responded more slowly overall $(2103 \pm 348 \mathrm{~ms})$ than controls $\left(1218 \pm 175 \mathrm{~ms} ; t_{(8.22)}=6.91 ; p=\right.$ 0.0001 ), but they did not differ from controls in the effect of syntactic complexity on reaction time $\left(t_{(8.14)}=0.90 ; p=\right.$ 0.39) (Fig. 2b).

\section{Atrophy in nonfluent PPA patients}

The most markedly atrophic region in nonfluent PPA patients was the left precentral gyrus and sulcus extending through the inferior frontal sulcus (IFS) to the dorsal part of the posterior inferior frontal gyrus (IFG). There was also volume loss in the left superior temporal gyrus (STG), superior temporal sulcus (STS), and middle temporal gyrus (MTG), and the right IFS (Fig. 3, bluegreen; Table 3).

Brain regions modulated by syntactic complexity in controls Brain regions modulated by syntactic complexity in controls (i.e., more active for noncanonical conditions than canonical conditions) included the left dorsal posterior IFG and adjacent IFS and insula, a less extensive set of homologous regions in the right hemisphere, and the left mid-posterior STS and adjacent MTG (Fig. 3, hot; Table 4).

There was overlap between regions modulated by syntactic complexity in controls and atrophic regions in nonfluent PPA patients in three brain areas: the left dorsal posterior IFG and adjacent IFS, a smaller homologous region in the right IFS, and the left mid-STS (Fig. 3).

\section{Changes in functional activity in nonfluent PPA patients}

In nonfluent PPA patients, bilateral temporal regions, but not frontal regions, were modulated by syntactic complexity (supplemental
Fig. 1, supplemental Table 1, available at www.jneurosci.org as supplemental material). In the masked whole-brain analysis of group differences in modulation by syntactic complexity, the left dorsal posterior IFG and adjacent IFS were modulated less by syntactic complexity in nonfluent PPA patients than in controls, as was a smaller homologous region in the right hemisphere (Fig. 4, cold; Table 5). There were no regions that were modulated significantly more by syntactic complexity in nonfluent PPA than in controls.

We further examined signal change as a function of condition in three left hemisphere ROIs located in anterior and posterior language areas as indicated in Figure 4: the left posterior IFC, left mid-STS, and left posterior STS. The ROI in the left posterior IFC was defined as the intersection of the regions modulated by syntactic complexity in controls and atrophic in nonfluent PPA, and was located in the left dorsal posterior IFG and adjacent IFS. This region was activated equivalently by all conditions in nonfluent PPA patients; modulation by syntactic complexity did not differ from zero $\left(t_{(7)}=-0.41, p=0.69\right.$ ) (Fig. $5 a$ ). However, overall activity in this region was marginally greater in patients than 
Table 5. Functional changes in nonfluent PPA patients

\begin{tabular}{|c|c|c|c|c|c|c|}
\hline \multirow[b]{2}{*}{ Contrast and brain region } & \multicolumn{3}{|c|}{$\begin{array}{l}\text { MNI } \\
\text { coordinates }\end{array}$} & \multirow{2}{*}{$\begin{array}{l}\text { Volume } \\
\left(\mathrm{mm}^{3}\right)\end{array}$} & \multirow[b]{2}{*}{ Maxt } & \multirow[b]{2}{*}{$p$} \\
\hline & $x$ & $y$ & $z$ & & & \\
\hline \multicolumn{7}{|c|}{ Modulated by syntactic complexity less in nonfluent PPA than in controls } \\
\hline Left dorsal posterior IFG and IFS & -44 & 21 & 25 & 1680 & 4.03 & $0.014^{\dagger}$ \\
\hline Right dorsal posterior IFG and IFS & 47 & 26 & 16 & 1336 & 5.87 & $0.027^{\dagger}$ \\
\hline \multicolumn{7}{|c|}{ Modulated by reaction time less in nonfluent PPA than in controls } \\
\hline Left IFG/S and precentral gyrus & -39 & 5 & 38 & 4072 & 4.07 & 0.007 \\
\hline \multicolumn{7}{|c|}{ Activated relative to rest more in nonfluent PPA than in controls } \\
\hline $\begin{array}{l}\text { Left anterior IFG (pars triangularis and } \\
\text { orbitalis) }\end{array}$ & -46 & 25 & 6 & 5664 & 6.17 & 0.003 \\
\hline \multicolumn{7}{|c|}{ Activated more in nonfluent PPA than controls, accuracy and reaction time matched } \\
\hline $\begin{array}{l}\text { Left anterior IFG (pars triangularis and } \\
\text { orbitalis) }\end{array}$ & -48 & 25 & 8 & 2560 & 6.34 & 0.045 \\
\hline
\end{tabular}

Search volume restricted to the set of regions activated in normal controls.

controls $\left(t_{(10.31)}=2.20, p=0.052\right)$. Note that a similar pattern of activity was also observed in the smaller homologous region in the right hemisphere posterior IFC that was likewise modulated by syntactic complexity in controls and atrophic in patients (supplemental Fig. 2, available at www.jneurosci.org as supplemental material).

In contrast, the left mid-STS (the other left hemisphere region that was modulated by syntactic complexity in controls and atrophic in patients) was modulated by syntactic complexity in nonfluent PPA patients $\left(t_{(7)}=2.95, p=0.022\right.$ ) (Fig. $5 b)$, and the extent of modulation did not differ between patients and controls $\left(t_{(15.25)}=0.05, p=0.96\right)$. The difference in modulation by syntactic complexity between this temporal ROI and the frontal ROI was greater in patients than in controls $\left(t_{(24.32)}=3.33, p=0.0028\right)$.

The ROI in the posterior STS was defined as the part of the temporal region that was modulated by syntactic complexity in controls but was not significantly atrophic in patients. This region patterned like the posterior IFC in that it was not modulated by syntactic complexity in nonfluent PPA patients $\left(_{(7)}=1.00\right.$, $p=0.35$ ) (Fig. $5 c$ ). In the ROI analysis (unlike the less powerful masked whole-brain analysis), this lack of modulation differed significantly from controls $\left(t_{(18.16)}=-2.84, p=0.011\right)$.

For the secondary contrast of interest-all conditions relative to rest-there were no regions that were significantly less active overall in nonfluent PPA patients than in controls. There was one region in which activity was greater in patients than controls: the left anterior IFG (Fig. 4, warm; Table 5). This region was centered at the border of the pars orbitalis and pars triangularis, and was located anterior and ventral to the posterior IFC region that showed reduced modulation by complexity in nonfluent PPA. To determine whether increased signal in the anterior IFG might be related to the marginally greater overall signal in the posterior IFC reported above, we examined the relationship between overall signal in these two regions. Overall signal was correlated between the anterior and posterior IFG in both patients $(r=0.85$, $p=0.0083)$ and controls $(r=0.51, p=0.011)$.

Within the nonfluent PPA group, extent of atrophy did not predict either modulation by syntactic complexity or overall activity in the posterior IFC, mid-STS, posterior STS, or anterior IFG (all $p$ values $\geq 0.15$ ).

\section{Ancillary functional analyses based on performance}

The first ancillary analysis used reaction time on correct trials as a proxy for syntactic complexity. Brain regions modulated by reaction time in controls were similar to those activated for nonca- nonical versus canonical conditions, but were more extensive (supplemental Table 2, available at www.jneurosci.org as supplemental material). In patients, frontal regions were not significantly modulated by reaction time (supplemental Table 3, available at www.jneurosci.org as supplemental material), and when the groups were compared directly, only left frontal regions were modulated less by reaction time in patients than controls, specifically the left IFG, adjacent IFS, and precentral gyrus (Fig. 6, cool; Table 5), similar to the region that was modulated less by the contrast of noncanonical and canonical conditions (compare Fig. 4 , cool). Modulation of the left posterior IFC ROI by reaction time in patients did not differ significantly from zero $\left(t_{(7)}=1.45\right.$, $p=0.19)$.

In the second ancillary analysis, we compared conditions that were matched for performance (accuracy and reaction time) between patients and controls. Even when performance was matched, the left anterior IFG showed greater activity in patients $(0.86 \pm 0.18 \%)$ than controls $\left(0.05 \pm 0.10 \% ; t_{(10.97)}=4.04, p=\right.$ 0.0020 ) (Fig. 6, warm; Table 5). This was the same region that showed more overall activity in patients than controls (compare Fig. 4, warm).

\section{Discussion}

In this study, we have shown that the atrophic left posterior IFC is not differentially recruited for the processing of syntactically complex sentences in nonfluent PPA. This region still responded to the syntactic processing task in nonfluent PPA patients; in fact, overall responses relative to rest were marginally greater than in controls. However there was an equivalent level of functional activity for noncanonical and canonical conditions. This pattern of activity differed significantly from the pattern observed in controls, in whom this region was functionally modulated by syntactic complexity.

The lack of functional modulation of the posterior IFC by syntactic complexity in nonfluent PPA cannot be accounted for solely in terms of differences in task performance between patients and controls (Price et al., 2006). Although reaction times were slower overall in patients, they were not disproportionately slower on noncanonical conditions. Under the assumption that reaction time reflects syntactic processing demands, we can conclude based on the reaction time data that patients made greater demands on syntactic processing resources for the more syntactically complex conditions just as controls did, but unlike controls, they did not recruit the posterior IFC as those demands increased. Consistent with this, the ancillary analysis restricted to correct trials showed that the posterior IFC was functionally modulated by reaction time in controls but not in patients.

It is also unlikely that our findings reflect merely hemodynamic changes associated with atrophy (D'Esposito et al., 2003), for two reasons. First, neither modulation of functional activity by complexity nor overall functional activity was correlated with tissue volumes in any region of interest. Second, the mid-STS was also atrophic, yet it continued to be modulated by syntactic complexity in patients just as it was in controls.

We propose two possible explanations for the lack of functional modulation by syntactic complexity in the posterior IFC in nonfluent PPA. The first is that cell death or cell damage in the posterior IFC renders this region unable to perform computations relevant to syntactic processing, and thus it is no longer recruited as syntactic processing demands increase. If this is the case, then the overall greater activity in the posterior IFC in patients must be interpreted as reflecting a separate mechanism not specific to syntactic processing. This explanation is supported by 

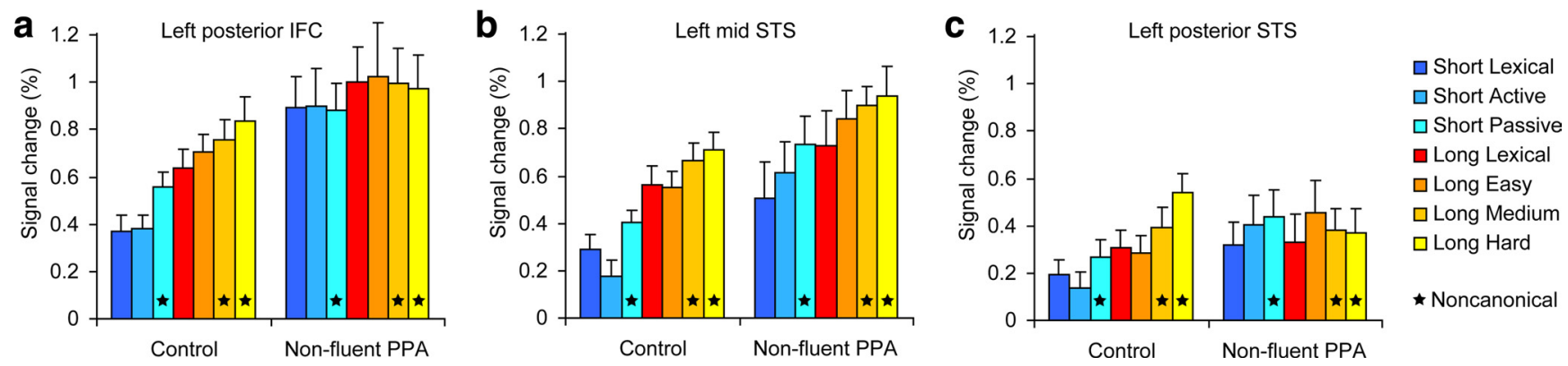

Figure 5. Signal change by condition in nonfluent PPA and in controls. Locations of ROIs are shown in Figure 4. $\boldsymbol{a}$, Signal in the posterior IFC was modulated by syntactic complexity in controls but not in patients. $\boldsymbol{b}$, Signal in the mid-STS region (that was atrophic in patients) was modulated by syntactic complexity equivalently in controls and patients. $\boldsymbol{c}$, Signal in the posterior STS region (that was not atrophic in patients) was modulated by syntactic complexity in controls but not in patients. Noncanonical conditions are indicated by the star symbol. Error bars show SEM. Summary measures of mean signal change in each ROl for canonical and noncanonical conditions in patients and controls are shown in supplemental Figure 3 (available at www.jneurosci.org as supplemental material).

the fact that overall activity in the posterior IFC was highly correlated with overall activity in the anterior IFG, which was over-recruited in patients, even when patients and controls were compared on conditions matched for accuracy and reaction time. Increased prefrontal activation has been observed in many imaging studies of normal aging as well as dementia and other neurological conditions, and is thought to reflect domain-general compensatory processes (Buckner, 2004; Park and Reuter-Lorenz, 2009). The kinds of domain-general processing performed by the anterior part of the IFG do not appear sufficient to support the domain-specific requirements of syntactic processing; therefore, patients performed poorly despite invoking a compensatory mechanism.

The second possible explanation is that the posterior IFC continues to have some syntactic functionality, but its efficiency is degraded such that even the easiest (canonical) conditions result in maximal recruitment and maximal signal in this region. Note that signal change for the easiest condition in patients was approximately equal to signal change for the most difficult condition in controls. Under this account, the compensatory activity in the anterior IFG could be interpreted as an anterior spread of activation from the posterior IFC, or as a qualitatively distinct domain-general compensatory process.

Previous studies of syntactic processing deficits in nonfluent PPA using structural (Amici et al., 2007, Peelle et al., 2008, Wilson et al., 2010) and functional (Cooke et al., 2003) imaging have also implicated left inferior frontal areas. The specific regions reported in these studies are quite variable and are located in or adjacent to both the posterior and anterior inferior frontal regions reported here. Our findings, which combine functional imaging data in both patients and controls as well as structural imaging data in patients, suggest that the anterior IFG has a more domain-general function, since its recruitment did not support successful syntactic processing, and that the posterior IFC is the most critical region underlying syntactic processing and its breakdown in nonfluent PPA. Specifically, the region that was modulated by complexity in controls, atrophic in patients, and not modulated by complexity in patients, was localized to the dorsal posterior IFG and adjacent IFS. Note that we observed atrophy to be most prominent in this region and more dorsally in the precentral gyrus and sulcus. The anterior insula showed only about $10 \%$ volume loss, and was thus less significantly atrophic than in some previous studies (e.g., Gorno-Tempini et al., 2004), which may reflect our use in the present study of a highdimensional normalization algorithm, DARTEL (Ashburner, 2007), which has less of a tendency than earlier methods to interpret widening of the Sylvian fissure as atrophy of the adjacent insula.

The other region that was modulated by syntactic complexity in controls and atrophic in nonfluent PPA was the mid-STS. In striking contrast to the posterior IFC, this region showed normal modulation by syntactic complexity in patients. Although atrophy in nonfluent PPA is most prominent in the dominant frontal lobe (Gorno-Tempini et al., 2004), temporal cortex is increasingly affected as the disease progresses (Rohrer et al., 2009). The patients we scanned were $2-10$ years post-onset, and so as a group had significantly reduced tissue volume in the left temporal lobe. Mid-posterior temporal regions have been implicated in syntactic processing in neuropsychological (Dronkers et al., 2004) and functional neuroimaging studies (e.g., Ben-Shachar et al., 2004; Bornkessel et al., 2005), and it has recently been suggested that the 
STS may be important for integrating lexical/semantic information with syntactic information (Friederici et al., 2009) or retrieving syntactically relevant information about lexical items (Snijders et al., 2009). Functions such as these may be differentially taxed in parsing syntactically complex sentences, because of an increased number of "references" to the lexical items contained in a sentence in the course of processing it. The preserved modulation of this region in nonfluent PPA implies that while the mid-STS may be necessary for syntactic processing, it is not sufficient, since task performance was poor. The normal activity in this region also underscores the specificity of the functional changes observed in the posterior IFC.

A more posterior sector of the STS was also modulated by syntactic complexity in controls. This region was not atrophic in nonfluent PPA, yet functionally it resembled the posterior IFC in that it was not modulated by complexity in patients. Unlike the mid-STS, which has been linked to ventral pathways associated with lexical and semantic functions (Saur et al., 2008), the posterior STS appears to be linked via dorsal tracts (the arcuate fasciculus and superior longitudinal fasciculus) to regions including the posterior IFC. This dorsal pathway has been argued to be important for syntactic processing (Friederici et al., 2006). The lack of functional modulation in the posterior STS suggests that dysfunction in nonfluent PPA is not restricted to the atrophic posterior IFC, but involves also this anatomically connected posterior language region.

Our study has several limitations, of which two are particularly notable. The first is that because PPA is not particularly common, we successfully scanned only eight patients with nonfluent PPA. These patients comprised a heterogeneous group, varying in stage of disease progression and degree of agrammatism. The relatively small number of patients studied precluded us from looking in more detail at relationships in individuals between atrophy, functional changes, and performance, and limited our ability to draw firm conclusions from the fact that we did not observe any significant correlations between atrophy and functional measures within the patient group. However, to our knowledge, this is still one of the larger cohorts of PPA patients diagnosed with a single variant to be studied with a functional activation paradigm, and we were still able to demonstrate robust functional differences between patients and controls.

A second notable limitation is that our syntactic processing task makes demands on other processes, such as working memory, attention, and executive function, which likely increase as syntactic complexity increases. Relatedly, there are different aspects to syntactic complexity that are usually correlated, such as syntactic movement, hierarchical complexity, verbal working memory, and reanalysis. Some researchers have attempted to distinguish between the neural correlates of these different aspects of syntactic complexity (Caplan and Waters, 1999; Grewe et al., 2005; Amici et al., 2007; Bahlmann et al., 2007; Peelle et al., 2008; Makuuchi et al., 2009), but we did not attempt to do so. Although we matched sentence length, lexical content, and point of disambiguation, thus ruling out superficial differences between conditions, our measure of complexity still encompasses several syntactic and nonsyntactic factors. Most functional imaging studies of syntactic processing have used similar designs that are subject to the same limitations. Despite these limitations, the syntactic processing regions that we identified in controls match well with regions that result in syntactic deficits when lesioned in patients (Bates et al., 1987a,b; Caplan and Hildebrandt, 1998; Dronkers et al., 2004).
In sum, we have shown that the atrophic left posterior IFC is not differentially recruited for the processing of syntactically complex sentences in nonfluent PPA, as it is healthy control subjects. This likely reflects either the complete lack of syntactic functionality of this region or degraded functionality such that the region is maximally recruited for even the simplest sentences. Thus the left posterior IFC is not only structurally abnormal, but also functionally compromised in nonfluent PPA, suggesting that this region plays a critical role in syntactic processing and in its breakdown in this syndrome.

\section{References}

Amici S, Brambati SM, Wilkins DP, Ogar J, Dronkers NL, Miller BL, GornoTempini ML (2007) Anatomical correlates of sentence comprehension and verbal working memory in neurodegenerative disease. J Neurosci 27:6282-6290.

Ashburner J (2007) A fast diffeomorphic image registration algorithm. Neuroimage 38:95-113.

Ashburner J, Friston KJ (2005) Unified segmentation. Neuroimage 26:839-851.

Bahlmann J, Rodriguez-Fornells A, Rotte M, Münte TF (2007) An fMRI study of canonical and noncanonical word order in German. Hum Brain Mapp 28:940-949.

Bates E, MacWhinney B (1989) Functionalism and the competition model. In: The cross-linguistic study of sentence processing (MacWhinney B, Bates E, eds), pp 3-73. Cambridge UK: Cambridge UP.

Bates E, Friederici A, Wulfeck B (1987a) Grammatical morphology in aphasia: evidence from three languages. Cortex 23:545-574.

Bates E, Friederici A, Wulfeck B (1987b) Comprehension in aphasia: a cross-linguistic study. Brain Lang 32:19-67.

Ben-Shachar M, Palti D, Grodzinsky Y (2004) Neural correlates of syntactic movement: converging evidence from two fMRI experiments. Neuroimage 21:1320-1336.

Bornkessel I, Zysset S, Friederici AD, von Cramon DY, Schlesewsky M (2005) Who did what to whom? The neural basis of argument hierarchies during language comprehension. Neuroimage 26:221-233.

Brainard DH (1997) The psychophysics toolbox. Spat Vis 10:433-436.

Buckner RL (2004) Memory and executive function in aging and AD: multiple factors that cause decline and reserve factors that compensate. Neuron 44:195-208.

Caplan D, Hildebrandt H (1998) Disorders of syntactic comprehension. Cambridge, MA: MIT Press.

Caplan D, Waters GS (1999) Verbal working memory and sentence comprehension. Behav Brain Sci 22:77-94.

Caplan D, Alpert N, Waters G (1999) PET studies of syntactic processing with auditory sentence presentation. Neuroimage 9:343-351.

Caramazza A, Zurif EB (1976) Dissociation of algorithmic and heuristic processes in language comprehension: evidence from aphasia. Brain Lang 3:572-582.

Cooke A, DeVita C, Gee J, Alsop D, Detre J, Chen W, Grossman M (2003) Neural basis for sentence comprehension deficits in frontotemporal dementia. Brain Lang 85:211-221.

Cotelli M, Borroni B, Manenti R, Ginex V, Calabria M, Moro A, Alberici A, Zanetti M, Zanetti O, Cappa SF, Padovani A (2007) Universal grammar in the frontotemporal dementia spectrum: evidence of a selective disorder in the corticobasal degeneration syndrome. Neuropsychologia 45:3015-3023.

D’Esposito M, Deouell LY, Gazzaley A (2003) Alterations in the BOLD fMRI signal with ageing and disease: a challenge for neuroimaging. Nat Rev Neurosci 4:863-872.

Dickerson BC, Sperling RA (2009) Large-scale functional brain network abnormalities in Alzheimer's disease: insights from functional neuroimaging. Behav Neurol 21:63-75.

Dronkers NF, Wilkins DP, Van Valin RD Jr, Redfern BB, Jaeger JJ (2004) Lesion analysis of the brain areas involved in language comprehension. Cognition 92:145-177.

Friederici AD, Bahlmann J, Heim S, Schubotz RI, Anwander A (2006) The brain differentiates human and non-human grammars: functional localization and structural connectivity. Proc Natl Acad Sci U S A 103:2458-2463.

Friederici AD, Makuuchi M, Bahlmann J (2009) The role of the posterior superior temporal cortex in sentence comprehension. Neuroreport 20:563-568. 
Friston KJ, Ashburner J, Kiebel S, Nichols T, Penny W, eds (2007) Statistical parametric mapping. London: Academic.

Gibson E (1998) Linguistic complexity: locality of syntactic dependencies. Cognition 68:1-76.

Goodglass H (1993) Understanding aphasia. San Diego: Academic.

Gorno-Tempini ML, Dronkers NF, Rankin KP, Ogar JM, Phengrasamy L, Rosen HJ, Johnson JK, Weiner MW, Miller BL (2004) Cognition and anatomy in three variants of primary progressive aphasia. Ann Neurol 55:335-346.

Gorno-Tempini ML, Hillis AE, Weintraub S, Kertesz A, Mendez M, Cappa SF, Ogar J, Rohrer JD, Black S, Boeve BF, Manes F, Dronkers N, Vandenberghe R, Rascovsky K, Patterson K, Miller BL, Knopman DS, Hodges JR, Mesulam M, Grossman M (2011) Classification of primary progressive aphasia and its variants. Neurology, in press.

Grewe T, Bornkessel I, Zysset S, Wiese R, von Cramon DY, Schlesewsky M (2005) The emergence of the unmarked: a new perspective on the language-specific function of Broca's area. Hum Brain Mapp 26:178-190.

Grossman M, Moore P (2005) A longitudinal study of sentence comprehension difficulty in primary progressive aphasia. J Neurol Neurosurg Psychiatry 76:644-649.

Grossman M, Rhee J, Moore P (2005) Sentence processing in frontotemporal dementia. Cortex 41:764-777.

Hodges JR, Patterson K (1996) Nonfluent progressive aphasia and semantic dementia: a comparative neuropsychological study. J Int Neuropsychol Soc 2:511-524.

Kramer JH, Jurik J, Sha SJ, Rankin KP, Rosen HJ, Johnson JK, Miller BL (2003) Distinctive neuropsychological patterns in frontotemporal dementia, semantic dementia, and Alzheimer disease. Cogn Behav Neurol $16: 211-218$

Makuuchi M, Bahlmann J, Anwander A, Friederici AD (2009) Segregating the core computational faculty of human language from working memory. Proc Natl Acad Sci U S A 106:8362-8367.

Mesulam MM (2001) Primary progressive aphasia. Ann Neurol 49:425-432.

Nestor PJ, Graham NL, Fryer TD, Williams GB, Patterson K, Hodges JR (2003) Progressive non-fluent aphasia is associated with hypometabolism centred on the left anterior insula. Brain 126:2406-2418.

Park DC, Reuter-Lorenz P (2009) The adaptive brain: aging and neurocognitive scaffolding. Annu Rev Psychol 60:173-196.

Peelle JE, Troiani V, Gee J, Moore P, McMillan C, Vesely L, Grossman M (2008) Sentence comprehension and voxel-based morphometry in pro- gressive nonfluent aphasia, semantic dementia, and nonaphasic frontotemporal dementia. J Neurolinguistics 21:418-432.

Pelli DG (1997) The VideoToolbox software for visual psychophysics: transforming numbers into movies. Spat Vis 10:437-442.

Price CJ, Crinion J, Friston KJ (2006) Design and analysis of fMRI studies with neurologically impaired patients. J Magn Reson Imaging 23:816-826.

Rabinovici GD, Jagust WJ, Furst AJ, Ogar JM, Racine CA, Mormino EC, O’Neil JP, Lal RA, Dronkers NF, Miller BL, Gorno-Tempini ML (2008) Abeta amyloid and glucose metabolism in three variants of primary progressive aphasia. Ann Neurol 64:388-401.

Rohrer JD, Warren JD, Modat M, Ridgway GR, Douiri A, Rossor MN, Ourselin S, Fox NC (2009) Patterns of cortical thinning in the language variants of frontotemporal lobar degeneration. Neurology 72:1562-1569.

Saur D, Kreher BW, Schnell S, Kümmerer D, Kellmeyer P, Vry MS, Umarova R, Musso M, Glauche V, Abel S, Huber W, Rijntjes M, Hennig J, Weiller C (2008) Ventral and dorsal pathways for language. Proc Natl Acad Sci U S A 105:18035-18040.

Snijders TM, Vosse T, Kempen G, Van Berkum JJ, Petersson KM, Hagoort P (2009) Retrieval and unification of syntactic structure in sentence comprehension: an FMRI study using word-category ambiguity. Cereb Cortex 19:1493-1503.

Stromswold K, Caplan D, Alpert N, Rauch S (1996) Localization of syntactic comprehension by positron emission tomography. Brain Lang 52:452-473.

Thompson CK, Ballard KJ, Tait ME, Weintraub S, Mesulam M (1997) Patterns of language decline in non-fluent primary progressive aphasia. Aphasiology 11:297-321.

Tyler LK, Marslen-Wilson W (2008) Fronto-temporal brain systems supporting spoken language comprehension. Philos Trans R Soc Lond B Biol Sci 363:1037-1054.

Weintraub S, Mesulam MM, Wieneke C, Rademaker A, Rogalski EJ, Thompson CK (2009) The northwestern anagram test: measuring sentence production in primary progressive aphasia. Am J Alzheimers Dis Other Demen 24:408-416.

Wertz RT, LaPointe LL, Rosenbek JC (1984) Apraxia of speech in adults: the disorder and its management. New York: Grune and Stratton.

Wilson SM, Isenberg AL, Hickok G (2009) Neural correlates of word production stages delineated by parametric modulation of psycholinguistic variables. Hum Brain Mapp 30:3596-3608.

Wilson SM, Henry ML, Besbris M, Ogar JM, Dronkers NF, Jarrold W, Miller BL, Gorno-Tempini ML (2010) Connected speech production in three variants of primary progressive aphasia. Brain 133:2069-2088. 